Pacific Journal of Mathematics

USING BRICK PARTITIONINGS TO ESTABLISH CONDITIONS
WHICH INSURE THAT A PLANO CONTINUUM IS A 2-CELL, 


\title{
USING BRICK PARTITIONINGS TO ESTABLISH CONDITIONS WHICH INSURE THAT A PEANO CON. TINUUM IS A 2-CELL, A 2-SPHERE OR AN ANNULUS
}

\section{RICHARD SLOCUM}

\begin{abstract}
Using brick patitioning, sufficient conditions are established for a subset of a Peano space to be locally euclidean. If $M$ is a Peano space with no local cut points and $S$ is a subcontinuum of $M$, has no local cut points, is the closure of a domain in $M$, has connected complement and contains a point $x$ such that every simple closed curve in $S$ not passing through $x$ separates $M$, then $S$ is a closed 2-cell, a 2-sphere or an annulus.
\end{abstract}

Three corollaries to the main theorem are started here. If $M$ is a Peano space with no local cut points and for each point $x \in M$, there is a neighborhood $U$ of $x$ such that every simple closed curve in $U-x$ separates $M$, then $M$ is a 2-manifold. If $M$ is a Peano space with no local cut points and, for some $m \geqq 1$, every disjoint union of $m$ simple closed curves separates $M$, then $M$ is a 2-manifold. If $M$ is a Peano continuum with no local cut points having a collection $C$ of $m(m \geqq 0)$ simple closed curves such that any simple closed curve in $M$ belongs to $C$ if and only if it does not separate $M$, then (1) $M$ is a 2-manifold with boundary $\cup C$ and (2) $M$ is a subspace of a 2-sphere.

The main theorem and first corollary are generalizations of theorems proved by Gail Young in [6].

The proof of the main theorem uses brick partitionings, the Kline sphere characterization [3], and the construction used in the proof of the Kline theorem to show that a certain set satisfies the conditions of Zippin's characterization of a closed 2-cell [5; page 92, Theorem 5.2]. R. H. Bing developed the concept of partitioning in [2] to solve the convex metric problem. Bing first proved the Kline theorem in [1]. He proved the Kline theorem again in [3] by using brick partitionings. When we speak of the Kline theorem in this paper, we shall speak of the form of the theorem in [3]. Thus the Kline theorem and the main theorem are closely related. The relationship is apparently best observed by the use of brick partitionings.

Several other corollaries to the main theorem are presented.

1. Preliminaries. For definitions of standard point set terms, the reader is referred to [5], while for terms concerning partitioning the reader is referred to [3].

We begin by making precise certain terms which are not universal. 
By a Peano space we mean a nondegenerate space which is metrizable, connected, locally connected and locally compact. A Peano continuum is a compact Peano space. Domain means connected open set. A 2-manifold is a connected metric space in which each point has a neighborhood homeomorphic to a closed 2-cell. The boundary of a 2-manifold is the set of points of a 2-manifold which do not have neighborhoods homeomorphic to an open 2-cell.

We conclude this section with the statements of three theorems with appropriate references given for their proofs. We say an arc $\alpha$ irreducibly separates a point $p$ from a point $q$ in $M$ if $\alpha$ separates $p$ from $q$ in $M$, but no proper subarc of $\alpha$ separates $p$ from $q$ in $M$.

Theorem 1.1 Let $M$ be a Peano continuum which is not separated by the omission of any two of its points and $\alpha$ an arc of $M$ with end points $p$ and $q$ which irreducibly separates a point $x$ from $a$ point $y$ in $M$. Then there exists a simple closed curve $J$ in $M$ which intersects $\alpha$ only in $p$ and $q$ while $M-J$ is connected and has property $S$.

Proof. The proof belongs to Bing [3; Theorem 20].

THEOREM 1.2. (Kline sphere characterization). If $M$ is a Peano continuum which is not separated by a pair of points, then either $M$ is a 2-sphere or there is a simple closed curve $J$ in $M$ such that $M-J$ is connected and has property $S$.

Proof. See [3; Theorem 20]. Theorem 1.1 implies Theorem 1.2.

THeorem 1.3. (L. Zippin). A Peano continuum Containing a simple closed curve $J$ and satisfying the following three conditions is a closed 2-cell with boundary $J$ :

(a) $C$ contains an arc which spans $J$.

(b) Every arc of $C$ that spans $J$ separates $C$.

(c) No proper closed subset of an arc spanning $J$ separates $C$.

Proof. See [5; page 92, Theorem 5.1].

2. Main theorem. One way to prove that a Peano space is a 2-manifold is to show that each point of the space has a closed 2-cell neighborhood. It is sufficient to show that each point has a neighborhood which is a 2-manifold. Such a neighborhood might be a closed 2-cell, a 2-sphere, or an annulus. The main theorem yields sufficient conditions which will insure that a set is a closed 2-cell, a 2-sphere, or an annulus.

Actually, the main theorem is really two theorems; the first theorem being a special case of the second theorem. It appears to be easier to prove the theorem for a special case first and then apply 
the special case to the proof of the more general case.

Each proof utilizes brick partitionings.

Lemma 2.1. Let $M$ be a Peano space with no local cut points. Suppose $D$ is a domain whose closure is a proper subset of $M$, is a Peano continuum, has connected complement and has the property that every simple closed curve in $\bar{D}$ separates $M$. Then $\bar{D}$ contains a simple closed curve $J$ such that $\bar{D}-J$ is connected and has property $S$, while $\operatorname{Bd} \bar{D} \subset J$.

Proof. Obviously $\bar{D}$ is not a 2-sphere. Thus by Theorem 1.2 there is a simple closed curve $J$ in $\bar{D}$ such that $\bar{D}-J$ is connected and has property $S$. Bd $\bar{D} \subset J$, since $J$ separates $M$.

We define the $E$ metric on $K$ for $x \in K, y \in K$ as $E(x, y)$ is the greatest lower bound of the diameter of all connected subsets of $K$ which contain $x$ and $y$. One problem which arises when one is dealing with a set with property $S$ occurs when one desires to partition the set in such a way that the boundary has certain properties. When this happens, one would rather deal with a set which is uniformly locally connected. But if a set has property $S$ and is connected and locally connected, it is uniformly locally connected under the $E$ metric. The $E$ metric also preserves the original topology.

Lemma 2.2. Suppose $D$ is a domain with property $S, \bar{D}$ has no local cut points and $\mathrm{Bd} D$ is a set with no isolated points. Then for every $\varepsilon>0$, there exists a brick partitioning $G=\left[g_{0}, g_{1}, \cdots, g_{n}\right]$ of $D$ such that $E\left(\bar{D} ; \mathrm{Bd} D, g_{i}\right) \geqq 0$, the equality holding if and only if $i \neq 0$, and for $i=1, \cdots, n$ the diameter of $g_{i}$ is less than $\varepsilon, \bar{g}_{0} \cap \bar{g}_{i} \neq \varnothing$, and $\bar{g}_{i} \cap \mathrm{Bd} D$ consists of more than one point.

Proof. The proof essentially belongs to Bing 13; Theorem 1]. For a complete proof see [4].

THeOREM 2.3. Let $M$ be a Peano space with no local cut points. Suppose $M$ contains a domain $D$ such that $\bar{D}$ is a Peano continuum with no local cut points and $M-\bar{D}$ is connected (or empty). Suppose also that every simple closed curve of $\bar{D}$ separates $M$.

Then $\bar{D}$ is either a closed 2-cell or a 2-sphere.

Proof. If $\bar{D}=M$, then $M$ is a 2-sphere by Theorem 1.2. If $D \neq M$, then, by Lemma $2.1, \bar{D}$ contains a simple closed curve $J$ such that $R=\bar{D}-J$ is connected and has property $S$, while $\operatorname{Bd} \bar{D} \subset J$.

Let $\varepsilon<1100$ diameter of $\mathrm{Bd} \bar{D}$. By Lemma 2.2 there is a brick 
partitioning $G=\left[g_{0}, \cdots, g_{n}\right]$ of $R$ such that $\bar{g}_{0} \cap J \neq \varnothing$ and for each $i=1, \cdots, n$ the diameter of each $g_{i}$ is less than $\varepsilon, \bar{g}_{i} \cap J$ consists of more than a point and $\bar{g}_{i} \cap \bar{g}_{0} \neq \varnothing$.

The remainder of this proof is given in sectionalized form. It will be convenient at times to denote $J$ by $J_{n+1}$.

2.31. Each $\bar{g}_{i}$ for $i=0,1, \cdots, n$ has connected complement in $M$.

Proof. For $i=0, M-\bar{g}_{0}=(M-R) \cup\left(\bigcup_{i=1}^{n}\left(\bar{g}_{i}-\mathrm{Bd} \bar{g}_{0}\right)\right)$ is a connected set. For $i \neq 0, M-\bar{g}_{i}$ is connected by the definition of brick partitioning and choice of $\varepsilon$.

2.32. Each $\bar{g}_{i}$ for $i=0,1, \cdots, n$ has a simple closed curve $J_{i}$ which does not separate $\bar{g}_{i}$, while $\operatorname{Bd} \bar{g}_{i} \subset J_{i}$.

Proof. Each $g_{i}$ has property $S$, hence each $\bar{g}_{i}$ has property $S$. Since for compact spaces property $S$ and local connectivity are equivalent properties, each $\bar{g}_{i}$ is locally connected. Thus each $\bar{g}_{i}$ is a Peano continuum. Note that no $\bar{g}_{i}$ is all of $M$. By Lemma 2.1 and 2.31, each $\bar{g}_{i}$ has a simple closed curve $J_{i}$ which does not separate $\bar{g}_{i}$, while $\mathrm{Bd} \bar{g}_{i} \subset J_{i}$.

2.33. If $i \neq j$ and $J_{i} \cap J_{j} \neq \varnothing$ for $i, j=0, \cdots, n+1$, then $J_{i} \cap J_{j}$ is an arc.

Proof. Suppose $i<j$ and $J_{i} \cap J_{j}$ is separated. Then there are at least two open arcs of $J_{i}-J_{j}$ which have end points on $J_{j}$. The properties of the brick partitioning $G$ make it possible to choose one such open arc, say $\alpha$, such that another such open arc contains a limit point of $\bar{D}-\bar{g}_{i}$. There are two arcs of $J_{j}$ which have the same end points as does $\alpha$. Again the properties of the brick partitioning $G$ make it possible to choose one of these arcs, say $\beta$, such that the other arc contains a limit point of $\bar{D}-\bar{g}_{j}$, if $j \neq n+1$, or a limit point of $\mathrm{Bd} \bar{D}$, if $j=n+1$. Then $\alpha \cup \beta$ forms a simple closed curve $J^{\prime}$.

If $j \neq n+1$, then from the properties of $G, \bar{D}-\left(\bar{g}_{i} \cup \bar{g}_{j}\right)$ is a connected set. Now $\bar{g}_{i}-J^{\prime}$ contains a limit point of $\bar{D}-\left(\bar{g}_{i} \cup \bar{g}_{j}\right)$ and $\bar{g}_{j}-J^{\prime}$ contains a limit point of $\left(\bar{D}-\left(\bar{g}_{i} \cup \bar{g}_{j}\right)\right) \cup\left(\bar{g}_{i}-J^{\prime}\right)$; hence $\bar{D}-J^{\prime}$ is connected. The diameter of $J^{\prime}$ is less than $2 \varepsilon$, thus

$$
\left(\bar{D}-J^{\prime}\right) \cap \operatorname{Bd} \bar{D} \neq \varnothing \text {. }
$$

Hence $M-J^{\prime}$ is connected. Thus $J_{i} \cap J_{j}$ is connected.

If $j=n+1, \bar{D}-J^{\prime}$ is connected since the closure of each brick meets $\bar{g}_{0}$. Also $\beta$ was chosen so that $\left(\bar{D}-J^{\prime}\right) \cap \operatorname{Bd} \bar{D} \neq \varnothing$. Thus $M-J^{\prime}$ is connected. Thus $J_{i} \cap J_{n+1}$ is connected.

By the properties of $G$, the intersection can not be either a point 
or a simple closed curve. Thus $J_{i} \cap J_{j}$ is an arc.

2.34. If $J_{i} \cap J_{j} \neq \varnothing i, j,=1, \cdots, n$, then $J_{i} \cap J_{j} \cap J$ consists of at most one point.

Proof. Let $x_{1}$ and $x_{2}$ denote the end points of the are $J \cap J_{i}$ and $y_{1}$ and $y_{2}$ denote the end points of arc $J \cap J_{j}$. Suppose $J_{i} \cap J_{j} \cap J$ consists of more than one point. Let $\beta$ denote the interior of the arc $J_{i} \cap J_{j} \cap J$.

Suppose some $J_{k}, k \neq i, j$ intersects $\beta$. The set $\left(J_{i}-\beta\right) \cup\left(J_{j}-\beta\right)$ contains a simple closed curve $J^{\prime}$. $J^{\prime}$ does not separate in $\bar{D}$ since, by the properties of the brick partitioning $G$, the closure of each brick of $G$ except $g_{i}$ and $g_{j}$ meets $J_{0}$ in points not on $J^{\prime}$, while $\bar{g}_{k}$ meets $\bar{g}_{i}$ and $\bar{g}_{j}$ in points not on $J^{\prime}$. By choice of $\varepsilon, J^{\prime}$ does not separate in $M$.

Suppose that $J_{i} \cap J$ and $J_{j} \cap J$ have an end point $x_{1}=y_{1}$ in common. Denote the open arc of $J_{i}-\left(J \cup J_{0}\right)$ with one end point $x_{1}$ and other end point $z_{1} \in J_{0}$ by $\alpha$. Denote the open arc of $J_{j}-\left(J \cup J_{0}\right)$ with one end point $x_{1}$ and the other end point $z_{2} \in J_{0}$ by $\gamma_{\text {. If }} z_{1} \neq z_{2}$, then choose $\lambda$ an arc of $J_{0}$ with end points $z_{1}$ and $z_{2}$ such that $J_{0}-\lambda$ meets some $\bar{g}_{k}$, where $g_{k} \in G$ and $k \neq 0, i, j$. Now $\alpha \cup \gamma \cup \lambda$ contains a simple closed curve $J^{\prime}$. By 2.33, the closure of each brick of $G$ except $g_{0}$ meets $J-J^{\prime}$ while $\bar{g}_{0}$ meets some $\bar{g}_{k}, k \neq 0, i, j$ in points of $J_{0}-J^{\prime}$. Hence $J^{\prime}$ does not separate in $M$. If $z_{1}=z_{2}$, then the closure of $J_{i}-J_{j}$ and $J_{j}-J_{i}$ form two arcs whose union is a simple closed curve $J^{\prime}$. Now $J \cap J^{\prime}$ is the arc of $J$ with end points $x_{2}$ and $y_{2}$. Also $J \cap J_{0}$ is an arc since $z_{1}=z_{2}$. The closure of every brick of $G$ except $g_{i}$ and $g_{j}$ meets the open arc $J_{0}-J^{\prime}$. Now $J-J^{\prime}$ is contained in the component of $\bar{D}-J^{\prime}$ which contains $J_{0}-J^{\prime}$. Thus $J^{\prime}$ does not separate in $M$.

Thus we may assume $y_{1}$ is an interior point of the arc $J \cap J_{i}$ and $x_{1} \notin J \cap J_{j}$. Denote the subarc of $J \cap J_{j}$ with end points $x_{1}$ and $y_{1}$ by $x_{1} y_{1}$. We also assume no $\bar{g}_{k} \cap J_{i}$ is a subset of $x_{1} y_{1}$. For if this is the case we may replace $g_{j}$ by some $g_{l} \in G$ for which these conditions do hold. Denote the open arc from $y_{1}$ to $J_{0}$ on $J_{i}-\left(J \cup J_{0}\right)$ by $\alpha$ with $z$ the end point of $\alpha$ on $J_{0}$. Start at $y_{1}$ and travel along $J \cap J_{i}$ to $x_{1}$, along $J_{i}-J$ to $J_{0}$, along $J_{0}$ to $z$ on an arc $\gamma$ such that $J_{0}-\gamma$ meets some $\bar{g}_{k}, k \neq i, j$, and along $\alpha$ to $y_{1}$ forming a simple closed curve $J^{\prime}$. Now $J^{\prime}$ does not separate in $\bar{D}$ since the closure of each brick of $G$ except $g_{0}$ meets the open arc $J-J^{\prime}$ while the closure of $g_{0}$ meets the closure of some $g_{k}$, where $k \neq i, j$. By the choice of $\varepsilon, J^{\prime}$ does not separate in $M$. Thus $J_{i} \cap J_{j} \cap J$ is a point.

2.35. $\bar{D}$ is a closed 2-cell with boundary $J$. 
Proof. We show that $\bar{D}$ satisfies conditions (a), (b), and (c) of Theorem 1.3.

(a) There is an arc spanning $J$.

Proof. $J_{1}-J$ is an open arc with distinct end points on $J$.

(b) Every arc spanning $J$ separates $\bar{D}$.

Proof. Suppose $x z y$ is an arc spanning $J$ which does not separate $\bar{D}$. Let $x w y$ denote one of the arcs of $J$ with end points $x$ and $y$ such that $J-x w y$ meets $\operatorname{Bd} \bar{D}$. The simple closed curve $J^{\prime}=x z y \cup$ $x w y$ separates a point $t$ from a point $s$ in $\bar{D}$. Since $\bar{D}$ is locally connected, we may choose $s$ and $t$ in $R$. Then there exists an arc tps in $\bar{D}-x z y$ from $t$ to $s$. Suppose the $\varepsilon$ chosen above for $G$ also satisfies $\varepsilon<\min \{d(t, J), d(s, J),(1 / 3) d(t p s, x z y)\}$. Note that $t$ and $s$ are elements of $g_{0}$.

For each $i=1, \cdots, n$ for which $t p s \cap \bar{g}_{i} \neq \varnothing$, let $\alpha_{i}$ denote the open $\operatorname{arc} J_{i}-J$. By 2.33, 2.34, and the choice of $\varepsilon$,

$$
\left(t p s \cap \bar{g}_{0}\right) \cup\left(\bigcup_{i} \alpha_{i}\right)=K
$$

is a connected subset of $\bar{D}-J^{\prime}$ joining $t$ to $s$. Then $J^{\prime}$ does not separate $t$ from $s$ in $\bar{D}$.

(c) No proper closed subset of an arc spanning $J$ separates $\bar{D}$.

Proof. Let $x z y$ be an arc spanning $J$. Suppose some proper closed subset $K$ of $x z y$ separates a point $p$ from $q$ in $\bar{D}$. We may choose $p$ and $q$ to be in $R$. Let $\alpha$ denote one of the $\operatorname{arcs}$ of $J$ with end points $x$ and $y$. Now $\alpha \cup K$ is contained in an arc $\lambda \subset \alpha \cup x z y$ which separates $p$ from $q$. Thus some subarc $\beta$ of $\lambda$ irreducibly separates $p$ from $q$. Since $\alpha$ does not separate space, one of the end points of $\beta$ belongs to $R$. By Theorem 1.1, there is a simple closed curve $J^{\prime}$ which does not separate $\bar{D}$ and intersects $\beta$ only at its end points. Since $\operatorname{Bd} \bar{D} \subset J^{\prime}$, there is a subarc $\gamma$ of $J^{\prime}$ spanning $J$. Thus $\gamma$ does not separate $\bar{D}$. But by part (b), $\gamma$ does separate space.

LEMmA 2.4. Let $K$ be any space with property $S$ which has no local cut points. No closure of any brick belonging to a brick partitioning of $K$ has any local cut points.

Proof. Each brick is uniformly locally connected under $E(K ; x, y)$.

The next lemma is a slight improvement of a theorem by Young [6; Theorem 2.3]. The proof here is new and, unlike Young's proof, uses brick partitioning. 
Lemma 2.5. Let $M$ be a Peano space with no local cut points. Then for every $\varepsilon>0$ and $p \in M$, there is a domain $D$ in $M$ having property $S$ such that $p \in D$, while $\bar{D}$ is a Peano continuum, has no local cut points, has connected complement and is of diameter less than $\varepsilon$.

Proof. Let $p \in M$. By [5; page 79, Theorem 3.7], $p$ has a uniformly locally connected neighborhood $U$ of diameter less than $\varepsilon$ such that $\bar{U}$ is a Peano continuum. By [5; page 82, Cor. 3.16[, $p$ has a neighborhood $U^{\prime}$ contained in $U$ such that $M-U$ is contained in a single component of $M-U^{\prime}$. By [3; Theorem 8], there is a brick partitioning $G$ of $\bar{U}$ such that each brick of $G$ is of diameter less than $(1 / 3) d\left(p, \mathrm{Bd} U^{\prime}\right)$. Let $D$ denote the maximal brick consolidation in $U^{\prime}$ which contains $p$ and is such that $\bar{D}$ is the union of the closures of the elements of $G$ which do not have a limit point in $\operatorname{Bd} U$.

Since $D$ is a brick, $D$ has property $S$. Thus $\bar{D}$ has property $S$. Since $\bar{D}$ is compact and has property $S, \bar{D}$ is lc; hence $\bar{D}$ is a Peano continuum. By Lemma 2.4, $\bar{D}$ has no local cut points.

We now show that $M-\bar{D}$ is connected. $\bar{D} \subset U^{\prime}$, hence $M-U$ is contained in a single component of $M-\bar{D}$. Since $D$ is maximal, every point of $U-\bar{D}$ lies in the closure of an element of $G$ which may be consolidated into a brick in $\bar{U}$ whose closure meets $\operatorname{Bd} U$.

Theorem 2.6. Let $M$ be a Peano space with no local cut points and $x^{\prime} \in M$. Suppose $x^{\prime}$ belongs to the closure of a domain $D$ such that $\bar{D}$ is a Peano continuum with no local cut points and $M-\bar{D}$ is connected (or empty). Suppose also that every simple closed curve of $\bar{D}$ not passing through $x^{\prime}$ separates $M$.

Then $D$ is a closed 2-cell, a 2-sphere, or an annulus.

Proof. Let $\delta<$ diameter Bd $\bar{D}$ if $\bar{D} \neq M$. By Lemma 2.5, for each $x^{\prime} \neq y \in \bar{D}, y$ has a neighborhood $G$ in $\bar{D}$ which is a Peano continuum with no local cut points, $x^{\prime} \notin G, \bar{D}-G$ is connected and the diameter of $G$ is less than $\delta$. Now every simple closed curve in $G$ separates $\bar{D}$ since the diameter of $\mathrm{Bd} \bar{D}$ is greater than $\delta$. By Theorem 2.3, $G$ is a closed 2-cell. Thus $\bar{D}-x^{\prime}$, is a 2-manifold.

Suppose $y \neq x^{\prime}$ belongs to a simple closed curve $J$ in $\bar{D}$ which does not separate $\bar{D}$. We now show that $y$ belongs to the manifold boundary of $\bar{D}-x^{\prime}$. Suppose $y$ is not a point of the manifold boundary. Then $y$ has a closed 2-cell neighborhood $G$ in $\bar{D}-x^{\prime}$ such that $\gamma=G \cap J$ is an arc while $G-\gamma$ consists of two components $C_{1}$ and $C_{2}$. Let $x \in C_{1} \cap$ Int $G$ and $z \in C_{2} \cap$ Int $G$. Let $\alpha$ be an arc from $x$ to $z$ in Int $G$ such that $\alpha \cap \gamma=y$. Now $\left(\bar{D}-x^{\prime}\right)-\operatorname{Bd} \bar{D}$ 
is a connected set. Denote the manifold boundary of $\bar{D}-x^{\prime}$ by $B$. We note that $N=\left(\bar{D}-x^{\prime}\right)-(\mathrm{Bd} \bar{D} \cup B)$ is also connected. Thus there is an arc $\beta$ in $N$ from $x$ to $z$. Now $\alpha \cup \beta$ contains a simple closed curve $J^{\prime}$ such that $J^{\prime} \cap J=y$. Since $J^{\prime}$ does not meet the manifold boundary of $\bar{D}-x^{\prime}, J^{\prime}$ has an annular neighborhood. Thus if $J^{\prime}$ does separate $\bar{D}$, there are two components of $\bar{D}-J^{\prime}$. But $J-y$ meets both sides of the annular neighborhood of $J^{\prime}$. So $J^{\prime}$ does not separate in $\bar{D}$.

There are two cases. We shall reduce the second case to the first case.

Case 1. Every simple closed curve in $\bar{D}-x^{\prime}$ separates $\bar{D}$.

Case 2. There is a simple closed curve $J_{1}$ in $\bar{D}-x^{\prime}$ which does not separate $\bar{D}$.

Suppose Case 2 holds. Then $\operatorname{Bd} \bar{D} \subset J_{1}$, for $J_{1}$ separates $M$. Also since we have shown that $J_{1}$ is contained in the manifold boundary of $\bar{D}-x^{\prime}, J_{1}$ is the only simple closed curve in $\bar{D}-x^{\prime}$, which does not separate $\bar{D}$. Consider the free union $C$ of $\bar{D}$ and a closed 2-cell $G$ under a homeomorphism from the manifold boundary of $G$ onto $J_{1}$. We note that $C$ is a Peano continuum with no local cut points and $C-x^{\prime}$ is a 2-manifold. Now any simple closed curve in $C-x^{\prime}$ which does not separate $C$ may be replaced by a simple closed curve in $\bar{D}-x^{\prime}$ which does not separate $C$; hence does not separate in $\bar{D}$. Thus every simple close curve in $C-x^{\prime}$ separates $C$.

If we think of $C$ as $\bar{D}$ and $C$ as $M$ in Case 1, we have only to deal with Case 1. Then if in Case 1, $\bar{D}$ can be shown to be a closed 2-cell or a 2-sphere, $\bar{D}$ in Case 2 is an annulus or closed 2-cell. We now deal with Case 1.

If $\bar{D}$ is not a 2 -sphere, then, by Theorem $1.2, \bar{D}$ contains a simple closed curve $J$ such that $R=\bar{D}-J$ is connected and has property $S$. Note that $x^{\prime} \in J$, since $J$ does not separate $\bar{D}$.

Let $\varepsilon<1 / 100$ diameter of $\operatorname{Bd} \bar{D}$ if $\bar{D} \neq M$, or if $\bar{D}=M$, let $\varepsilon<$ $1 / 100$ diameter of $J$. By Lemma 2.2, there is a brick partitioning $G=\left[g_{0}, \cdots, g_{n}\right]$ of $R$ such that $\bar{g}_{0} \cap J=\varnothing$ and for each $i=1, \cdots, n$ the diameter of each $g_{i}$ is less than $\varepsilon, \bar{g}_{i} \cap J$ consists of more than one point and $\bar{g}_{i} \cap \bar{g}_{0} \neq \varnothing$.

Now $\left(\bar{D}-x^{\prime}\right)-g_{0}$ is connected since each brick of $G$ except $g_{0}$ has closure which meets $J$. By Lemma 2.4, $\bar{g}_{0}$ has no local points. Thus by Theorem 2.3, letting $M=\bar{D}-x$ and $D=g_{0}, \bar{g}_{0}$ is a closed 2-cell.

This also proves that $\mathrm{Bd} \bar{D} \subset J$. For if $y \in \mathrm{Bd} \bar{D} \cap R$, then $\varepsilon$ may be chosen so that $y \in g_{0}$. Since $\bar{g}_{0}$ is a closed 2-cell and $y$ is not a local 
cut point of $M$, one may construct a simple closed curve $J^{\prime}$ in $g_{0}$ $x^{\prime}-y$ which separates $\bar{g}_{0}$ into two components each of which intersects Bd $\bar{D}$. Then $J^{\prime}$ does not separate in $M$.

That $\bar{D}$ is a closed 2-cell with boundary $J$ follows from Theorem 1.3 .

(a) There is an arc spanning $J$.

Proof. Let $x, y, z \in J$ be such that $\left\{x, x^{\prime}\right\}$ separates $y$ from $z$ on $J$. There is an arc $\alpha$ from $y$ to $z$ in $\bar{D}-\left\{x, x^{\prime}\right\}$. Then $\alpha \cap R \neq \varnothing$. By using the order topology on $\alpha$, one easily obtains a subarc of $\alpha$ which spans $J$.

(b) Every arc spanning $J$ separates $\bar{D}$.

Proof. Let $\alpha$ be an arc spanning $J$ with $z \in \alpha \cap R$. Choose $\varepsilon$ so that $z \in g_{0}$. Let $G$ be a closed 2-cell neighborhood of $z$ in $\bar{g}_{0}$ such that $\beta=G \cap \alpha$ is an arc and $G-\beta$ consists of exactly two components $C_{1}$ and $C_{2}$ such that $\bar{C}_{1}$ and $\bar{C}_{2}$ are both closed 2-cells. Let $r \in C_{1}$ and $t \in C_{2}$. Suppose $\alpha$ does not separate $r$ from $t$ in $\bar{D}$. Then there is an arc $\gamma$ from $r$ to $t$ in $\bar{D}-\alpha$ which also misses $x^{\prime}$. Since $J-x^{\prime}$ is contained in the manifold boundary of $\bar{D}-x^{\prime}, \gamma$ can be replaced by an arc $\gamma^{\prime}$ lying in $R$ such that $\gamma^{\prime}$ joins $r$ to $t$ in $\bar{D}-\alpha$. Choose $\varepsilon$ so $\varepsilon<d\left(\gamma^{\prime}, J\right)$. Denote the new $\bar{g}_{0}$ by $T$. Obviously, $T$ is a closed 2-cell, $\gamma^{\prime} \subset T$, and the old $\bar{g}_{0} \subset T$. Let $\alpha^{\prime}$ denote the subarc of $\alpha$ containing $\beta$ which spans $T$. We have shown that $r$ is not separated from $t$ in $T$ by $\alpha^{\prime}$.

(c) No proper closed subset of an arc spanning $J$ separates $\bar{D}$.

Proof. Suppose $x z y$ is an arc spanning $J$. Suppose some proper closed subset $K$ of $x z y$ separates a point $p$ from $q$ in $\bar{D}$. We may choose $p$ and $q$ to be in $R$. Let $\gamma$ denote one of the arcs of $J$ with end points $x$ and $y$. Now $\gamma \cup K$ is contained in an arc $\lambda \subset \gamma \cup x z y$ which separates $p$ from $q$. Thus some subarc $\alpha$ of $\lambda$ irreducibly separates $p$ from $q$. Now one of the end points of $\alpha$, say $r$, is in $R$. Choose $\varepsilon$ so that $\varepsilon<d(r, J)$. Then $r \in g_{0}$. Let $G$ be a closed 2-cell neighborhood of $r$ such that $G \subset g_{0}$ and $G \cap \alpha=\beta$ and arc with one end point $r$ and other end point $t$. But $\beta$ does not separate $G$, hence the subarc of $\alpha$ with end points $s$ and $t$ separates $p$ from $q$ in $\bar{D}$. But $\alpha$ was irreducible with respect to that property.

3. Characterizations of 2-manifolds. The next result is an improvement of Young's characterization of 2-manifolds [6; Theorem 1.1], in that for each point $x$ of the space one needs only to find a neighborhood such that every simple closed curve in that neighbor- 
hood not passing through $x$ separates. One should note that Young's theorem may be obtained as a corollary from Theorem 2.3 via Lemma 2.5 .

Theorem 3.1. Let $M$ be a Peano space with no local cut points. Suppose that for each point $x \in M$, there is a neighborhood $U$ of $x$ such that every simple closed curve in $U-x$ separates $M$. Then $M$ is a 2-manifold.

Proof. By Lemma 2.5, each point $x \in M$ has a neighborhood $\bar{D}$ contained in $U$ such that $\bar{D}$ is a Peano continuum, has no local cut points and has connected complement in $M$. Since $\bar{D} \subset U$ every simple closed curve in $\bar{D}-x$ separates $M$. By Theorem 2.6, $\bar{D}$ is a closed 2-cell, a 2-sphere, or an annulus. Since $\bar{D}$ is a neighborhood of $x, x$ has a closed 2-cell neighborhood.

Young obtained his characterization of 2-manifolds by first proving the following theorem. See [6; Theorem 3.1]. Note that Theorem 2.6 is a generalization of this theorem.

Theorem 3.2. If the Peano continuum $M$ has no local cut points, but contains a point $x$ such that every simple closed curve not passing through $x$ separates $M$, then $M$ is either a 2-sphere or a closed 2-cell.

Proof. This is the special case in which $\bar{D}=M$ in Theorem 2.6. Obviously, $M$ is not an annulus.

The following theorem is not a characterization of 2-manifolds. However, the converse of the theorem is well known for compact 2-manifolds. Thus we may consider the theorem a characterization of compact 2-manifolds. The usefulness of Theorem 2.6 is demonstrated in the proof of this theorem.

Theorem 3.3. Let $M$ be a Peano space with no local cut points. Suppose for some $m \geqq 1$ that every disjoint union of $m$ simple closed curves separate $M$. Then $M$ is a 2-manifold.

Proof. We first prove the existence of a collection of $m$ disjoint simple closed curves of $M$. Choose $m$ distinct points of $M$ and let $\varepsilon$ be less than one-half the minimum distance between any two of the points. By Lemma 2.5 each of these $m$ points has a neighborhood of diameter less than $\varepsilon$, such that each of these neighborhoods is a Peano continuum with no local cut points. It is well known that each of these neighborhoods has the property that every two points of the neighborhood are contained in a simple closed curve in that neighborhood. See [5; page 85, Theorem 3.32]. Thus each neighborhood 
contains a simple closed curve, and we have shown the existence of a disjoint collection of $m$ simple closed curves.

Choose $m$ so that $m$ is minimal with respect to the property stated above. If $m=1$, then $M$ is a 2-sphere by Theorem 1.2. Suppose $m>1$. Then there is a disjoint collection $K$ of $m-1$ simple closed curve $S_{1}, S_{2}, \cdots, S_{m-1}$ whose union does not separate $M$. Then every simple closed curve in $M-\mathrm{U} K$ separates $M$. Letting $M-\mathrm{U} K$ be both the $U$ and $M$ of Theorem $3.1 M-\mathrm{U} K$ is a 2-manifold.

Let $y \in S_{i} 1 \leqq i \leqq m-1$. Suppose $S_{i}$ may be replaced in $K$ by a simple closed curve $S_{i}^{\prime}$ where $y \notin S_{i}^{\prime}$ and such that $K^{\prime}$, the set formed by replacing $S_{i}$ by $S_{i}^{\prime}$ in $K$, is also a disjoint collection of $m-1$ simple closed curves whose union does not separate $M$. Again, by Theorem 3.1, $M-\mathrm{U} K^{\prime}$ is a 2-manifold. Thus $y$ has a closed 2-cell neighborhood.

Suppose every $S_{i}^{\prime}$ which replaces $S_{i}$ in $K$ passes through $y$. By Lemma 2.5, $y$ has a neighborhood $G$ such that for $i \neq j, G \cap S_{j}=\varnothing$, $G$ is a Peano continuum with no local cut points, and $M-G$ is connected. Now every simple closed curve in $G-y$ separates $M$. Thus, by Theorem 2.6, $G$ is a closed 2-cell, a 2 -sphere, or an annulus. Then $y$ also has a closed 2-cell neighborhood. Then $M$ is a 2-manifold.

One is also interested in knowing what Peano continua may be embedded in 2-spheres. The previous theorem is helpful because we can in many cases show first that a Peano continuum in a 2-manifold and then show that the 2-manifold is homeomorphic to a subspace of a 2-sphere.

THeorem 3.4. Let $M$ be a Peano continuum with no local cut points which for some integer $n \geqq 0$ has a collection $C=\left\{J_{1}, \cdots, J_{n}\right\}$ of $n$ simple closed curves such that any simple closed curve in $M$ belongs to $C$ if and only if it does not separate $M$. Then

(1) $M$ is a 2-manifold with boundary $\mathrm{U} C$, and

(2) $M$ is a subspace of a 2-sphere.

Proof. The proof will be done by induction on the number of elements in $C$. For $n=0, M$ is a 2 -sphere with empty boundary by Theorem 1.2. Suppose that the theorem is true for any space satisfying the conditions of the theorem where $C$ has $n$ elements. Let $M$ be a space satisfying the conditions of the theorem where $C$ has $n+1$ elements. By Theorem 3.3, $M$ is a 2-manifold. Let $T$ be closed 2cell with boundary $J$ and $h: J \rightarrow J_{1}$ a homeomorphism. Let $M^{\prime}$ be the disjoint union of $M$ and $T$ obtained by identifying each $x \in J$ with $h(x) \in J_{1}$. Note that $M^{\prime}$ is also a 2-manifold and $M$ is a subspace of $M^{\prime}$.

Suppose $M^{\prime}$ contains a simple closed curve $J^{\prime}$ which intersects $T$ 
but does not separate $M^{\prime}$. Since $M^{\prime}$ is a 2-manifold, $J^{\prime}$ may be altered near $T$ to form a new simple closed curve $J^{\prime \prime}$, which does not intersect $T$, does not separate $M^{\prime}$, and agrees with $J^{\prime}$ near each of $J_{2}, \cdots, J_{n+1}$. But $J^{\prime \prime}$ does not separate $M$ either. Hence $J^{\prime} \in J_{1}, \cdots, J_{n+1}$.

Thus $M^{\prime}$ satisfies the conditions of the theorem for the collection $C^{\prime}=\left\{J_{2}, \cdots, J_{n+1}\right\}$. By the induction hypothesis, $M^{\prime}$ is a 2-manifold with boundary $\cup C^{\prime}$ and $M^{\prime}$ is a subspace of a 2 -sphere $S$. Since $M$ is a subspace of $M^{\prime}, M$ is a subspace of $S$ and has manifold boundary $U C$.

As a corollary we have the following characterization of an annulus. It is also true that by considering a disjoint union with a closed 2-cell the following theorem is a direct corollary of Theorem 2.6.

THEOREM 3.5. Let $M$ be a Peano continuum with no local cut points and which has precisely two simple closed curves $J_{1}$ and $J_{2}$ which do not separate $M$. Then $M$ is an annulus.

Proof. Every disjoint union of three simple closed curves separates $M$. Thus by Theorem 3.4, $M$ is a 2-manifold with manifold boundary $J_{1} \cup J_{2}$ and is also a subspace of a 2-sphere.

4. Almost 2-manifolds. We shall say that a Peano space $P$ is an almost 2-manifold if $P$ contains a domain $D$ which is a 2-manifold and $\bar{D}=P$. In particular, we shall consider the case where $P$ is a Peano space with no local cut points, $x \in P$, and $D=P-x$ is a 2manifold. The reason for the interest in this case arises from the proof of Theorem 2.6. The proof essentially consists of showing that $\bar{D}$ is a 2-manifold knowing that $\bar{D}-x^{\prime}$ is a 2-manifold. The question of just how badly does a space need to behave in order that it fail to be a 2-manifold because of a singleton point is answered in this section.

Almost 2-manifolds may also arise by considering quotient spaces. If $M$ is a space and $J$ a simple closed curve of that space, let $M / J$ denote the quotient space obtained by identifying $J$ to a point.

Theorem 4.1. Let $M$ be a Peano continuum which contains a simple closed curve $J$ such that $M-J$ is connected, every simple closed curve in $M-J$ separates $M$ and $M / J$ has no local cut points. Then $M / J$ is a 2-sphere or a closed 2-cell.

Proof. Let $f: M \rightarrow M / J$ be the natural map and $f(J)=x$. Since every simple closed curve in $M / J-x$ separates $M / J$, by Theorem 3.2, $M / J$ is a 2 -sphere or a closed 2-cell.

Theorem 4.2. Suppose that $M$ is a Peano space with no local 
cut points which contains a point $x$ such that $M-x$ is a 2-manifold but $M$ is not. Then there is a sequence $\left\{J_{n}\right\}$ of disjoint simple closed curves in $M-x$ which converge to $x$ such that $\bigcup_{n}\left\{J_{n}\right\}$ does not separate $M$.

Proof. By Lemma 2.5, $x$ has neighborhood $D_{1}$ such that $\bar{D}_{1}$ is a Peano continuum with no local cut points, $M-\bar{D}_{1}$ is connected and the diameter of $\bar{D}_{1}$ is less than 1 . Since $\bar{D}_{1}$ is not a 2 -sphere, a closed 2-cell or an annulus, by Theorem 2.6, there is a simple closed curve $J_{1}$ in $\bar{D}_{1}-x$ which does not separate $M$. Let $M_{1}=M-J_{1}$ Again, by Lemma 2.5, $x$ has a neighborhood $D_{2}$ such that $\bar{D}_{2} \subset D_{1}, \bar{D}_{2}$ is a Peano continuum with no local cut points, $M_{1}-\bar{D}_{2}$ is connected, $J_{1} \cap D_{2}=\varnothing$, and the diameter of $\bar{D}_{2}$ is less than $1 / 2$. Again, by Theorem 2.6, there is a simple closed curve $J_{2}$ in $D_{2}-x$ which does not sepapate $M_{1}$. Let $M_{2}=M_{1}-J_{2}$. In this manner a sequence $\left\{J_{n}\right\}$ of simple closed curves, a sequence $\left\{\bar{D}_{n}\right\}$ of Peano continua and a sequence $\left\{M_{n}\right\}$ of Peano continua are obtained such that for each $n>$ 2, $D_{n}$ is a neighborhood of $x, \bar{D}_{n} \subset D_{n-1}, \bar{D}_{n}$ is a Peano continuum with no local cut points, $M_{n-1}-\bar{D}_{n}$ is connected, $\bar{D}_{n} \cap J_{n-1}=\varnothing, \bar{D}_{n}$ contains a simple closed curve $J_{n}$ such that $J_{n}$ does not separate $M_{n-1}, M_{n}=M_{n-1}-J_{n}$ and the diameter of $\bar{D}_{n}<1 / n$. Then the $\left\{J_{n}\right\}$ converges to $x$.

Let $K=\bigcup_{n}\left\{J_{n}\right\}$. Suppose $y$ and $z$ lie in different components of $M-K$. Then either $y=x$ or $z=x$ since, if not, then $y$ and $z$ for some $n$ would belong to $M_{n-1}-\bar{D}_{n}$. Then if $M-K$ is separated, $x$ would form a component of $M-K$. Thus $M-K$ is connected.

\section{REFERENCES}

1. R. H. Bing, The Kline sphere characterization problem, Bull. Amer. Math. Soc., 25 (1946), 644-653.

2. - Partitioning a set, Bull. Amer. Math. Soc., 55 (1949), 1101-1110.

3. - Complementary domains of continuous curves, Fund. Math., 36 (1949), 303318.

4. R. A. Slocum, Deriving Characterizations of 2-manifolds using brick partitionings, Dissertation Tulane University, (1969).

5. R. L. Wilder, Topology of manifolds, Amer. Math. Soc. Colloq. Pub. 32 (1949), Amer. Math. Soc.

6. Gail S. Young, A characterization of 2-manifolds, Duke Math. J., 14 no. 4, December, 1947.

Received September 21, 1970 and in revised form June 2, 1972.

The University of Tennessee at Martin 



\section{PACIFIC JOURNAL OF MATHEMATICS}

\section{EDITORS}

\section{H. SAMELSON}

Stanford University

Stanford, California 94305

\section{R. HOBBY}

University of Washington

Seattle, Washington 98105

\section{J. DugundjI}

Department of Mathematics University of Southern California Los Angeles, California 90007

RICHARD ARENS

University of California

Los Angeles, California 90024

\section{ASSOCIATE EDITORS}
E. F. BECKENBACH
B. H. NeumanN
F. WOLF
K. YOSHIDA

\section{SUPPORTING INSTITUTIONS}

\author{
UNIVERSITY OF BRITISH COLUMBIA \\ CALIFORNIA INSTITUTE OF TECHNOLOGY \\ UNIVERSITY OF CALIFORNIA \\ MONTANA STATE UNIVERSITY \\ UNIVERSITY OF NEVADA \\ NEW MEXICO STATE UNIVERSITY \\ OREGON STATE UNIVERSITY \\ UNIVERSITY OF OREGON \\ OSAKA UNIVERSITY
}

\author{
UNIVERSITY OF SOUTHERN CALIFORNIA \\ STANFORD UNIVERSITY \\ UNIVERSITY OF TOKYO \\ UNIVERSITY OF UTAH \\ WASHINGTON STATE UNIVERSITY \\ UNIVERSITY OF WASHINGTON \\ AMERICAN MATHEMATICAL SOCIETY \\ NAVAL WEAPONS CENTER
}

The Supporting Institutions listed above contribute to the cost of publication of this Journal, but they are not owners or publishers and have no responsibility for its content or policies.

Mathematical papers intended for publication in the Pacific Journal of Mathematics should be in typed form or offset-reproduced, (not dittoed), double spaced with large margins. Underline Greek letters in red, German in green, and script in blue. The first paragraph or two must be capable of being used separately as a synopsis of the entire paper. The editorial "we" must not be used in the synopsis, and items of the bibliography should not be cited there unless absolutely necessary, in which case they must be identified by author and Journal, rather than by item number. Manuscripts, in duplicate if possible, may be sent to any one of the four editors. Please classify according to the scheme of Math. Rev. Index to Vol. 39. All other communications to the editors should be addressed to the managing editor, Richard Arens, University of California, Los Angeles, California, 90024.

50 reprints are provided free for each article; additional copies may be obtained at cost in multiples of 50 .

The Pacific Journal of Mathematics is published monthly. Effective with Volume 16 the price per volume (3 numbers) is $\$ 8.00$; single issues, $\$ 3.00$. Special price for current issues to individual faculty members of supporting institutions and to individual members of the American Mathematical Society: $\$ 4.00$ per volume; single issues $\$ 1.50$. Back numbers are available.

Subscriptions, orders for back numbers, and changes of address should be sent to Pacific Journal of Mathematics, 103 Highland Boulevard, Berkeley, California, 94708.

PUBLISHED BY PACIFIC JOURNAL OF MATHEMATICS, A NON-PROFIT CORPORATION

Printed at Kokusai Bunken Insatsusha (International Academic Printing Co., Ltd.), 270, 3-chome Totsuka-cho, Shinjuku-ku, Tokyo 160, Japan. 


\section{Pacific Journal of Mathematics}

\section{Vol. 42, No. $3 \quad$ March, 1972}

Catherine Bandle, Extensions of an inequality by Pólya and Schiffer for vibrating membranes ................................ 543

S. J. Bernau, Topologies on structure spaces of lattice groups.......... 557

Woodrow Wilson Bledsoe and Charles Edward Wilks, On Borel product measures .......................................

Eggert Briem and Murali Rao, Normpreserving extensions in subspaces of

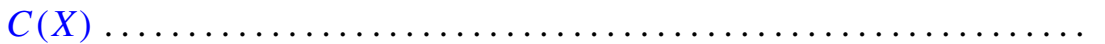

Alan Seymour Cover, Generalized continuation.................. 589

Larry Jean Cummings, Transformations of symmetric tensors .......... 603

Peter Michael Curran, Cohomology of finitely presented groups .......... 615

James B. Derr and N. P. Mukherjee, Generalized quasicenter and

hyperquasicenter of a finite group ...................... 621

Erik Maurice Ellentuck, Universal cosimple isols .................. 629

Benny Dan Evans, Boundary respecting maps of 3-mainfolds .......... 639

David F. Fraser, A probabilistic method for the rate of convergence to the

Dirichlet problem .................................. 657

Raymond Taylor Hoobler, Cohomology in the finite topology and Brauer

groups ..................................... 667

Louis Roberts Hunt, Locally holomorphic sets and the Levi form ........ 681

B. T. Y. Kwee, On absolute de la Vallée Poussin summability............ 689

Gérard Lallement, On nilpotency and residual finiteness in semigroups .... 693

George Edward Lang, Evaluation subgroups of factor spaces........... 701

Andy R. Magid, A separably closed ring with nonzero torsion pic ....... 711

Billy E. Rhoades, Commutants of some Hausdorff matrices ............. 715

Maxwell Alexander Rosenlicht, Canonical forms for local derivations . . . . 721

Cedric Felix Schubert, On a conjecture of L. B. Page ................ 733

Reinhard Schultz, Composition constructions on diffeomorphisms of $S^{p} \times S^{q}$

J. P. Singhal and H. M. (Hari Mohan) Srivastava, A class of bilateral generating functions for certain classical polynomials ....

Richard Alan Slocum, Using brick partitionings to establish conditions which insure that a Peano continuum is a 2-cell, a 2-sphere or an annulus...

James F. Smith, The p-classes of an $H^{*}$-algebra ...

Jack Williamson, Meromorphic functions with negative zeros and positive

poles and a theorem of Teichmuller ................. 